\title{
Molecular and Genetic Testing in Thrombosis and Hemostasis
}

\author{
David John Rabbolini, BSc, MBBCh, PhD, FRACP, FRCPA ${ }^{1,2}$ Maha Othman, MD, MSc, PhD ${ }^{3,4}$ \\ ${ }^{1}$ Lismore Base Hospital, Lismore Cancer Care and Haematology Unit, \\ Lismore Base Hospital, New South Wales, Australia \\ ${ }^{2}$ Northern Clinical School and the Rural Clinical School (Northern \\ Rivers), Faculty of Medicine and Health, University of Sydney, \\ Sydney, Australia \\ ${ }^{3}$ Department of Biomedical and Molecular Sciences, School of \\ Medicine, Queen's University, Kingston, Canada \\ ${ }^{4}$ School of Baccalaureate Nursing, St. Lawrence College, Kingston, \\ Canada \\ Semin Thromb Hemost 2019;45:657-660.
}

We are in a period of modern medicine where the focus of treatment approaches is shifting to specific molecular features of the patient and their disease. This era of "precision medicine" is geared toward the individual and moves beyond a "one-size fits all" approach that is determined by the phenotypic features of a disorder, with the ultimate goal that a detailed understanding of disease at a molecular level will enable targeted intervention and better clinical outcomes. $^{1}$

The advancement of molecular technologies and, in particular, genetic sequencing technologies have been instrumental tools in making precision medicine possible. Clinicians and scientists in the field of thrombosis and hemostasis have embraced the opportunity to incorporate molecular technology into research design and clinical practice. This has led to the emergence of these techniques into the clinic and their endorsement by many professional societies into guidelines and diagnostic algorithms. ${ }^{2,3}$ An understanding of current and emerging genetic testing strategies and their implementation is therefore of utmost importance to scientists, specialists, and practitioners in this area. In this special issue of Seminars in Thrombosis and Hemostasis, we have invited contributors to present reviews on key topics in molecular and genetic testing. As editors, we have attempted to provide readers with reviews that describe fundamental technologies, definitions, and principles in the field; use and updates on current molecular methods across various disorders of primary and secondary hemostasis; their implementation in the clinic; and finally translational developments.

Many researchers and clinicians may be familiar with basic genetic buzzwords and phrases but may not have a

Address for correspondence David John Rabbolini, BSc, MBBCh, PhD, FRACP, FRCPA, Lismore Cancer Care and Haematology Unit, 70-72 Hunter street, Lismore, NSW, 2480, Australia (e-mail: david. rabbolini@sydney.edu.au). clear understanding of their origins or synthesis. The first review covered in this special issue by Kumar et $\mathrm{al}^{4}$ focuses on the evolution of analytical platforms from their origins in Sanger sequencing ${ }^{5}$ capable of resequencing individual DNA templates, to next generation sequencing (NGS; second and third generation) capable of massively parallel sequencing. The authors discuss and define key elements of these platforms, as well as complementary and emerging strategies, aimed to improve sequence output and fidelity. This article is followed by another core paper by Webster and Hampshire ${ }^{6}$ that provides a seamless transition from analytical and prebioinformatic processes (as discussed by Kumar et $\mathrm{al}^{4}$ ) into the next phase of a molecular diagnostic pipeline, namely, bioinformatics and insilico prediction tools. Here, the authors provide detailed descriptions and analysis of currently available tools available to researchers that aid in variant annotation.

The main body of this issue is comprised of three reviews that describe the state of molecular diagnosis, the achievements, and current limitations in the setting of inherited platelet number and functional disorders, von Willebrand disease (VWD), and rare bleeding disorders. In the first of these reviews by Mumford and Westbury ${ }^{7}$ the authors focus on considerations of molecular testing in the context of inherited platelet disorders. Megakaryopoiesis is coordinated by a tightly regulated number of genes and transcription factors that act in a combinatorial manner producing platelets of adequate size and number, ${ }^{8,9}$ moreover, their function is a result of a simultaneous response to complex activator and opposing inhibitory signals, yet the traditional approach to diagnose these conditions relies on a step-wise
Copyright $\odot 2019$ by Thieme Medical Publishers, Inc., 333 Seventh Avenue, New York, NY 10001, USA. Tel: +1(212) 584-4662.
DOI https://doi.org/ 10.1055/s-0039-1694993. ISSN 0094-6176.
Genetic Testing in Thrombosis and Hemostasis; Guest Editors: David John Rabbolini, BSc, MBBCh, PhD, FRACP, FRCPA, and Maha Othman, MD, MSc, PhD. 
application of phenotypic tests that interrogate linear pathways of development and activation. ${ }^{10,11}$ The ability to diagnose these conditions, therefore, has often fallen short. ${ }^{11}$ Mumford and Westbury ${ }^{7}$ review the advantages of a molecular diagnosis in these conditions and critically appraise the use of gene panels, whole exome sequencing (WES), and whole genome sequencing (WGS) approaches noting an evolution toward WGS approaches into the diagnostic arena that provides the only practical approach to detect all potentially relevant variants including those in regulatory noncoding regions not assessed by the other platforms.

The next review by Bastida et $\mathrm{al}^{12}$ assesses the role of molecular testing across inherited bleeding disorders including, inherited platelet disorders and abnormalities of coagulation proteins. Focusing on conditions of coagulation proteins, the authors indicate that sensitive and simple routine coagulation assays are still best as first line tests to determine an abnormality in these proteins. Here, genetic testing provides an advantage by enabling determination of carrier status for individuals suspected of an abnormality in which coagulation assays may not always be clear-cut. Moreover, and importantly, the establishment of genotype-phenotype relationship enables greater understanding of bleeding patterns and provides prognostic information that may inform preventative bleeding strategies in some of these disorders.

The next review by Batlle et $\mathrm{al}^{13}$ assesses molecular testing in VWD. Currently, diagnosis is achieved using a well delineated set of blood tests that assess antigen level (von Willebrand factor: antigen VWF:Ag), activity (VWF: ristocetin cofactor [VWF:RCo] and VWF: collagen binding [VWF:CB]) and factor eight (FVIII) levels. A quantitative or qualitative abnormality of VWF is, in most cases, discernable by interpreting results and ratios generated by these assays. As indicated by Batlle et al, in some cases, additional tests, such as VWF:FVIII binding (VWF:FVIIIB), ristocetininduced platelet agglutination (RIPA), VWF propeptide, types I/III collagen binding, and VWF multimeric analysis are required to differentiate a particular functional defect of VWF. Molecular testing is generally reserved to distinguish cases of type-2N VWD versus hemophilia A, type-2B VWD versus platelet-type VWD, and in some cases of type- 3 disease. ${ }^{14,15}$ Unfortunately, as in other areas of hemostasis testing, this seemingly logical step-wise wise approach is hindered by preanalytical variables, test standardization, availability, and the need for trained personnel for result interpretation. ${ }^{13}$ Batlle et al describe molecular alternatives including a Sanger-based approach, which like the phenotypic approach is step-wise, complex, and costly but is sensitive and specific and, therefore (to date), has been considered the reference standard for molecular diagnosis in VWD. ${ }^{16}$ Authors comment on results of large Spanish and European studies that evaluate the diagnostic potential of NGS. ${ }^{13}$ These studies have generated an impressive amount of data on the nature of causative variants, and have been successful in identifying and/or reclassifying individuals with/without VWD. However, it is clear that while a NGS approach may eliminate many preanalytical problems inherent in a phenotypic approach, the need for the establishment of specialist centers to support a predominant NGS is essential.

The issue then provides three reviews that describe the incorporation of molecular methods in the hemostasis clinic. In a noteworthy article, Crispin and $\mathrm{Koo}^{7}$ discuss important practical and institutional ethical considerations surrounding the provision of a molecular diagnosis and describe how molecular tests are currently being employed in clinical practice to inform diagnostic, clinical decision making, and treatment approaches for individuals across disorders of hemophilia, rare bleeding disorders, platelet disorders, and thrombotic disorders. This review is followed by a provocative report by Favaloro ${ }^{18}$ who assesses the testing patterns of two common low-risk thrombophilia inherited genetic variants, factor $\mathrm{V}$ Leiden (G1691A) and the prothrombin gene G20210A mutation as performed at a large tertiary institution. The data presented demonstrate the accessibility of these genetic tests to referring physicians in everyday clinical practice. It further highlights, by comparing the incidence of these variants detected in various patients groups with their prevalence in the general population, that, in many cases, tests are requested inappropriately, in so much as the result does not influence management and may instead contribute to unwarranted psychological distress in patients with an otherwise probable low-risk venous thromboembolic events. Taken together, this article along with Crispin and $\mathrm{Koo}^{7}$ reinforce the need to exercise discretion, when considering pursuing genetic testing in the clinical arena. Yamaguti-Hayakawa and Ozelo ${ }^{19}$ provide the third article in this section, describing the ability to provide personalized care through molecular methods. Here, authors report on the tremendous advances that have contributed to the realization of an era of gene therapy in hemophilia. Recombinant wild-type adenoassociated virus (AAV) has been the most widely studied vector in this field. ${ }^{19}$ This nonintegrating viral vector is designed to deliver single stranded or self-complementary F9 gene, in the case of hemophilia B, or single-stranded B domain deleted $F 8$ gene in the case of hemophilia $A$, into transfected target cells with resultant increase in transgene expression, and an anticipated increase in protein secretion needed to support hemostasis. ${ }^{19}$ The authors set out the progress of this technology from preclinical trials through to human studies and detail hurdles that stand as obstacles to the efficacy of this delivery system. These include loss of transgene expression (due to the nonintegrating nature of AAV), host humoral, and cellular immune responses directed against the viral proteins, as well as questions relating to incorporation of gene therapies into special patient groups, such as children and patients with underlying liver diseases. Undoubtedly, the genesis of gene-editing strategies into clinical trials (NCT02695160) that employ zinc-finger nucleases, transcription activator-like effector nucleases (TALENs), and clustered regularly interspaced short-palindromic repeat 
(CRISPR)-Cas nucleases will catalyze ${ }^{20}$ our hope for cure in hemophilia.

The final review is written by Ghorbanpour and Lillicrap. $^{21}$ This paper closes the issue and steps away from technologies that are currently employed in the clinic and discusses exciting translational methods of chromatin conformation assays, liquid biopsies, and single-cell transcriptome analysis. Crucial elements in generating patientspecific genetic data are determining the significance of the generated molecular detail and adjudicating the influence (if any) from other intra or intergenic variants on the clinical phenotype. Authors describe the chromatin conformation assay that has potential in unraveling this conundrum. This technology uses the principle that DNA enhancer sequences present at remote sites from the transcriptional start site of a target gene derive their activating function through long-range chromatin interactions with proximal regulatory sequences. ${ }^{22}$ These interactions may be altered in the presence of a pathogenic sequence variant causing a disruption in chromatin conformation which is recorded. ${ }^{23}$ Next the prospect of liquid biopsy is reviewed with a particular focus on tumoreducated platelets. This platform utilizes the "plasticity" of the platelet transcriptome, which makes it potentially qualitatively different in health and disease. By analyzing platelet RNA and embracing machine learning classification algorithms, one is able to determine the presence, and type of cancer with a high degree of accuracy. ${ }^{24}$ Ghorbanpour and Lillicrap ${ }^{21}$ remind us that this field is still in its infancy but certainly by judging from its use in studies of unprovoked venous thrombosis (the PLATO study-ClinicalTrials. gov; identifier: NCT02739867) holds much potential. Finally, the authors conclude the article with a description of single-cell transcriptome analysis highlighting the potential of this technology to analyze rare cell populations within large pools of diverse cells. These platforms have already shown promise in studies of hematopoiesis ${ }^{25}$ and its extension into other areas of thrombosis and hemostasis is eagerly anticipated.

The contributions to this special issue addressing molecular and genetic testing in thrombosis and hemostasis are timely and comprehensive, and we hope the readership enjoys the articles.

\section{Conflicts of Interest}

None declared.

\section{References}

1 Yates LR, Seoane J, Le Tourneau C, et al. The European Society for Medical Oncology (ESMO) precision medicine glossary. Ann Oncol 2018;29(01):30-35

2 Gresele P; Subcommittee on Platelet Physiology of the International Society on Thrombosis and Hemostasis. Diagnosis of inherited platelet function disorders: guidance from the SSC of the ISTH. J Thromb Haemost 2015;13(02):314-322

3 Gomez K, Laffan M, Keeney S, Sutherland M, Curry N, Lunt P. Recommendations for the clinical interpretation of genetic variants and presentation of results to patients with inherited bleeding disorders. A UK Haemophilia Centre Doctors' Organisation good practice paper. Haemophilia 2019;25(01): 116-126

4 Kumar KR, Cowley MJ, Davis RL. Next generation sequencing and emerging technologies. Semin Thromb Hemost 2019;45(07): 661-673

5 Sanger F, Nicklen S, Coulson AR. DNA sequencing with chainterminating inhibitors. Proc Natl Acad Sci U S A 1977;74(12): 5463-5467

6 Webster SJAM, Hampshire DJ. A bioinformatics toolkit: in silico tools and online resources for investigating genetic variation. Semin Thromb Hemost 2019;45(07):674-684

7 Mumford AD, Westbury SK. Genetic techniques used in the diagnosis of inherited platelet disorders. Semin Thromb Hemost 2019;45(07):685-694

8 Pimkin M, Kossenkov AV, Mishra T, et al. Divergent functions of hematopoietic transcription factors in lineage priming and differentiation during erythro-megakaryopoiesis. Genome Res 2014;24(12):1932-1944

9 Wilson NK, Foster SD, Wang X, et al. Combinatorial transcriptional control in blood stem/progenitor cells: genome-wide analysis of ten major transcriptional regulators. Cell Stem Cell 2010;7(04): 532-544

10 Bye AP, Unsworth AJ, Gibbins JM. Platelet signaling: a complex interplay between inhibitory and activatory networks. J Thromb Haemost 2016;14(05):918-930

11 Gresele P, Harrison P, Bury L, et al. Diagnosis of suspected inherited platelet function disorders: results of a worldwide survey. J Thromb Haemost 2014;12(09):1562-1569

12 Bastida JM, Benito R, Lozano ML, et al. Molecular diagnosis of inherited coagulation and bleeding disorders. Semin Thromb Hemost 2019;45(07):695-707

13 Batlle J, Pérez-Rodríguez A, Corrales I, et al; PCM-EVW-ES Investigators Team. Update on molecular testing in von Willebrand disease. Semin Thromb Hemost 2019;45(07):708-719

14 Laffan MA, Lester W, O'Donnell JS, et al. The diagnosis and management of von Willebrand disease: a United Kingdom Haemophilia Centre Doctors Organization guideline approved by the British Committee for Standards in Haematology. Br J Haematol 2014;167(04):453-465

15 Castaman G, Goodeve A, Eikenboom J; European Group on von Willebrand Disease. Principles of care for the diagnosis and treatment of von Willebrand disease. Haematologica 2013;98 (05):667-674

16 Baronciani L, Goodeve A, Peyvandi F. Molecular diagnosis of von Willebrand disease. Haemophilia 2017;23(02):188-197

17 Crispin P, Koo RM. Genetic and molecular testing in thrombosis and hemostasis: informing surveillance, treatment and prognosis. Semin Thromb Hemost 2019;45(07):720-729

18 Favaloro EJ. Genetic testing for thrombophilia related genes: Observations of testing patterns for factor V Leiden (G1691A) and the prothrombin gene "mutation" (G20210A). Semin Thromb Hemost 2019;45(07):730-742

19 Yamaguti-Hayakawa GG, Ozelo MC. Gene therapy: Paving new roads in the treatment of hemophilia. Semin Thromb Hemost 2019;45(07):743-750

20 Cornu TI, Mussolino C, Cathomen T. Refining strategies to translate genome editing to the clinic. Nat Med 2017;23(04): 415-423

21 Ghorbanpour E, Lillicrap D. Innovative molecular testing strategies for adjunctive investigations in hemostasis and thrombosis. Semin Thromb Hemost 2019;45(07):751-756

22 Vernimmen D, Bickmore WA. The hierarchy of transcriptional activation: from enhancer to promoter. Trends Genet 2015;31 (12):696-708 
23 Barutcu AR, Fritz AJ, Zaidi SK, et al. C-ing the genome: a compendium of chromosome conformation capture methods to study higherorder chromatin organization. J Cell Physiol 2016;231(01):31-35

24 Best MG, Sol N, Kooi I, et al. RNA-seq of tumor-educated platelets enables blood-based pan-cancer, multiclass, and molecular pathway cancer diagnostics. Cancer Cell 2015;28 (05):666-676

25 Psaila B, Heuston EF, Milojkovic D, et al. A single-cell approach to unraveling abnormal megakaryocyte differentiation and function in myelofibrosis. Blood 2017;130(Suppl 1):490 